\title{
Oral Biopharmaceutics Tools: What is New and What is Coming? APV/FIP Joint-Workshop in Nuremberg
}

Sandra Klein*

Ernst Moritz Arndt University Greifswald Department of Pharmacy

Institute of Biopharmaceutics and Pharmaceutical Technology, Center of Drug Absorption and Transport Greifswald, Germany
e-mail:Sandra.Klein@uni-greifswald.de
D issolution testing is an important tool from early development to life cycle management of a drug product. With the aim to give an update on the application of dissolution testing in assessing critical formulation attributes with regard to bioavailability of the respective drug product, on September 26-27, 2017, on the initiative of Dr. Horst-Dieter Friedel who is cochair of the International Pharmaceutical Federation (FIP) Focus Group on Dissolution/In Vitro Drug Release, the International Association for Pharmaceutical Technology (APV) and FIP organized a joint workshop on oral biopharmaceutic tools in Nuremberg. The workshop was planned by Dr. Jennifer Dressman, the chair of the APV Focus Group on Biopharmaceutics and Pharmacokinetics, and Dr. Sandra Klein, also representing APV, and, Dr. Christos Reppas and Dr. Horst-Dieter Friedel from the FIP Focus Group on Dissolution/In Vitro Drug Release. The workshop had a total of 57 attendees.

Day 1 of the workshop was dedicated to the presentation of new approaches in biorelevant/biopredictive in vitro models. After a general introduction by Dr. HorstDieter Friedel, Bayer AG, Berlin, the scientific lectures started with a presentation given by Dr. Cord Andreas from the University of Frankfurt. He introduced the biopharmaceutics risk assessment roadmap (BioRAM) concept, which is intended to optimize drug product development and performance by using therapy-driven target drug delivery profiles as a framework to achieve the desired therapeutic outcome. The BioRAM concept considers the therapeutic target with the drug substance characteristics and enables collection of critical knowledge for development of a dosage form that can perform consistently for meeting the patient's needs. The presentation highlighted how key factors are identified and how in vitro, in vivo, and in silico modeling and simulation techniques are used to elucidate the optimal drug delivery rate and pattern.

The next speaker was Dr. Uwe Münster from Bayer AG, Germany, who gave an overview of the goals and activities of the OrBiTo project, an IMI initiative dedicated to a better understanding of oral drug absorption and using this knowledge for developing new biopharmaceutics in vitro and in silico tools that prospectively should help to reduce the number of preclinical and clinical studies required for new drug formulations but also could be applied in a regulatory framework, e.g., in the registration process of generic products. He presented results from work performed in the different work packages of the project and discussed future objectives.

Dr. Peter Langguth from the University of Mainz, Germany then presented on how to establish an in vitro in vivo relationship (IVIVR) or correlation (IVIVC). He introduced various strategies for designing predictive in vitro test methods and showed their implementation by sharing resultsfrom differentcasestudies. Healso nicely presented the chances and pitfalls with different in vitro models and clearly pointed out that a mechanistic understanding of the in vivo behavior of the dosage form is essential for obtaining an IVIVC for a given drug formulation. In the final part of his presentation he presented results from a survey performed in the OrBiTo project to get an overview of how IVIVC is established in industry and what are the biggest hurdles in trying to establish biopredictive in vitro models. He concluded that the chances of a successful IVIVC increase with the application of in vivo biopredictive dissolution methodologies and alternative approaches like physiologically based pharmacokinetic (PBPK) modeling would allow successful IVIVC development for drugs with more complicated absorption/disposition behavior.

The next presentation was given by Dr. Christos Reppas from the University of Athens, Greece, who first discussed the different interpretations of what is a biorelevant dissolution method and then introduced in detail the different physiological and dosage form-related parameters to be addressed in a biorelevant dissolution experiment. He explained in detail the different levels of simulation of luminal composition and how much complexity is required for adequately screening a certain formulation. He connected this question with the developability classification system (DCS) and highlighted the limits when the aim is to predict drug solubility in 
human aspirates based on results obtained in solubility experiments with biorelevant media.

The last presentation of the first day was given by Dr. Kerstin Frank from Boehringer Ingelheim, Germany. She talked about dissolution methodologies used in preclinical testing and how these can be guided by API properties, results from other early stage preclinical tests, and dosage form characteristics. Major criteria discussed were the selection of different apparatus or media types. She also presented two case studies where several of the discussed dissolution models were applied to prototype formulations containing BCS class II compounds. She concluded that in early stage drug development, close interdisciplinary cooperation is needed and that it is further important to identify critical drug/formulation attributes as early as possible to develop the right in vitro test methods.

The second day of the workshop started with a presentation from David Turner from Certara, UK. He gave a comprehensive introduction to the use of PBPK models and detailed information of how different PBPK models are designed, how they have advanced over the last years by implementing physiological data of different populations, intraluminal fluid dynamics, transporter expression along the GI tract, etc., and finally, how they can be applied to obtain a physiologically based IVIVC.

Dr. Cord Andreas then gave a talk on the prediction of in vivo performance of modified release (MR) dosage forms using biorelevant dissolution. With results from a case study examining drug release of an MR formulation containing a BCS class II compound with compendial and biorelevant test methods, he could clearly show the importance of properly addressing the changing environment in the different sections of the GI tract when the aim is to obtain an IVIVC for such a formulation. In another case example, he nicely showed how in vitro and in silico approaches can be combined to obtain a meaningful IVIVC for a highly soluble drug.

Dr. David Sperry from Eli Lilly, USA then presented an industrial view on the prediction of in vivo performance using biorelevant dissolution. He gave an insight into the application of biorelevant media and models in the pharmaceutical industry. Cases examples included the application of two-step dissolution models for poorly soluble weak bases, the application of the focused beam reflectance measurement (FBRM) to track particles in the dissolution vessel for better understanding the dissolution process, and the main determinants of dissolution rate after disintegration of a solid dosage form. He also highlighted the importance of the composition of the dissolution medium when the aim is to establish an IVIVC and discussed both the chances and limits of in-house models and commercially available in vitro and in silico PBPK software for predicting in vivo release performance of solid oral dosage forms. At the end of his presentation, he presented an interesting work flow applied for bioavailability risk assessment and underscored the importance of the detailed information that is needed on product performance in such a procedure.

The session on novel oral biopharmaceutics tools was completed by a presentation of Dr. Sheila Annie Peters from Merck KGaA, Germany. She presented industrial case examples on PBPK modeling. A focus of her presentation was how to verify PBPK models to establish confidence and how to perform a sensitivity analysis and an uncertainty analysis to demonstrate robustness of the model. In agreement with the statements made in previous talks, she pointed out that the proper verification of a model requires a detailed mechanistic understanding of dosage form performance and that currently there is still a big gap in knowledge in the complex interplay of parameters that are involved the essential processes determining the plasma profile of a drug after its oral administration.

The second session of day 2 of the workshop was dedicated to quality control (QC) applications. Dr. Anita Nair from Merck KGaA, Germany, was the first speaker of this session and presented quality by design ( $Q b D)$ applications of critical process parameters. She explained the elements of $Q b D$ starting with the quality target profile for a product through identifying critical quality attributes and critical material and process attributes to developing a control strategy by defining specifications for materials, product, and unit processes that would ensure quality of the product. She highlighted what role properly specified dissolution test methods play in this context when they are regarded as critical quality attributes.

Dr. Lorenz Liesum, Novartis, Switzerland then reported of how process analytical technology (PAT) tools and surrogate model development can be implemented in the pharmaceutical industry and how this fits with the FDA vision of control strategy. He clearly pointed out critical factors with regard to drug and excipient properties and manufacturing process that can be of either wellknown or random origin and can impact dissolution of the drug product. Moreover, he presented an interesting case example in which after applying a specific validated mathematical model, dissolution of a given product was nicely predicted by using critical process attributes. 
However, he also clearly pointed out the preconditions for using such models in a prospective manner and showed the limits for their application.

Prof. Dr. Christos Reppas closed the session on QC applications with a talk on linking QC methodology with biowaivers and bridging studies. A particular focus of this presentation was set on dissolution test conditions to be applied for a BCS-based biowaiver and how to translate the BCS biowaiver test method into a QC method.

The last session of the workshop was dedicated to regulatory opportunities and the objective was to provide global perspectives on the role of dissolution testing on product design, QC, and approval. Dr. Arzu Selen, US FDA was the first speaker and presented the FDA perspective on how in vitro and in vivo drug product performance can be connected. She discussed the role that dissolution testing has played in drug product development and QC, and she highlighted the progress that dissolution methodologies have undergone to become more and more biopredictive. She further discussed the potential impact of predictive methods and presented possible approaches for clinically relevant specifications for drug dissolution/release and spent also some time discussing the BioRAM strategy as an integrated systems approach to drug development. She concluded that advanced dissolution testing can impact candidate selection, drug product design, and development as well as drug product life cycle management. Dr. Selen also pointed out that a proper mechanistic understanding is essential for developing such models and that novel dissolution testing approaches, novel systems approaches, and utilization of IVIVC and IVIVR are highly encouraged.

Dr. J. Michael Morris, formerly HPRA, Ireland, shared the EU perspective on the use of dissolution tests. He also stated that dissolution testing is a useful development tool to provide information for formulation development and manufacture process optimization and that a properly designed dissolution method can be a surrogate for in vivo bioequivalence. The focus of his presentation was set on pharmacopoeial requirements and information provided in ICH Q6A and Q4B guidelines. Dr. Morris also discussed the requirements for dissolution test methods of novel dosage forms.

The final presentation was given by Dr. Tomokazu Tajiri, Astellas, Japan, who presented the Japanese perspectives on the role of dissolution testing in development. He discussed the harmonization of dissolution methodologies in the Japanese (JP), United States (USP) and European Pharmacopoeia (EP), but also pointed out that there are still some significant differences between JP and USP, and EP. He also highlighted that for dissolution test design, particularly for experiments simulating gastric conditions, the high prevalence of achlorhydria in Japan is currently an important parameter to consider when designing dissolution methods. The prevalence of achlorhydria in the Japanese population is also reflected in the recommendation to not use enzymes in dissolution media since at higher gastric $\mathrm{pH}$, the conversion of pepsinogen into pepsin will not be possible. A marked difference can also be observed in the number of dissolution test experiments to be performed at various test conditions that are required for registering a drug product. In addition to this, to date, the biowaiver concept is not applied in Japan, and the regulatory authorities do not accept IVIVC approaches.

In summary, the workshop highlighted the importance of oral biopharmaceutical tools in the development of safe and effective oral dosage forms. The presentations underlined the progress in vitro dissolution testing and in silico modeling has made during the past decades and provided the participants with a deep insight into the development of physiologically based in vitro and in silico models and the importance of interdisciplinary approaches. It was also of great importance to hear the regulatory viewpoints in Europe, Japan, and the United States and realize that there is a chance for a paradigm shift. The participants made use of their opportunity to discuss with all the speakers and hopefully gathered a lot of new ideas to be implemented to improv the predictivity and robustness of current in vitro and in silico models. 